\title{
Convergence rate of extremes from Maxwell sample
}

\section{Chuandi Liu* and Bao Liu}

\section{"Correspondence:}

liuchuandi@swu.edu.cn

School of Mathematics and

Statistics, Southwest University,

Chongqing, 400715, China

\begin{abstract}
For the partial maximum from a sequence of independent and identically distributed random variables with Maxwell distribution, we establish the uniform convergence rate of its distribution to the extreme value distribution.
\end{abstract}

MSC: Primary 62E20; 60E05; secondary 60F15; 60G15

Keywords: extreme value distribution; maximum; Maxwell distribution; uniform convergence rate

\section{Introduction}

One interesting problem in extreme value theory is to consider the convergence rate of some extremes. For the uniform convergence rate of extremes under the second-order regular variation conditions, see Falk [1], Balkema and de Haan [2], de Haan and Resnick [3] and Cheng and Jiang [4]. For the extreme value distributions and their associated uniform convergence rates for given distributions, see Hall and Wellner [5], Hall [6], Peng et al. [7], Lin and Peng [8] and Lin et al. [9].

In this note, we discuss the uniform convergence rate of extremes from a sequence of independent and identically distributed (iid) random variables with Maxwell distribution (MD). The probability density function of MD is given by

$$
f(x)=\sqrt{\frac{2}{\pi}} \frac{x^{2}}{\sigma^{3}} \exp \left(-\frac{x^{2}}{2 \sigma^{2}}\right), \quad x>0 .
$$

The MD and the convergence rate of extremes from Maxwell sample have been widely used in the field of physics. We establish the uniform convergence rate of its distribution to the extreme value distribution and give an improved proof for the pointwise convergence rate of MD.

Throughout this paper, let $\left(\xi_{n}, n \geq 1\right)$ be a sequence of iid random variables with common distribution $F(x)=\int_{0}^{x} f(t) d t$ with a probability density function $f(x)$ given by (1.1), and let $M_{n}=\max _{1 \leq k \leq n} \xi_{k}$ be the partial maximum. Liu and Fu [10] proved that

$$
\lim _{n \rightarrow \infty} P\left(\alpha_{n}^{-1}\left(M_{n}-\beta_{n}\right) \leq x\right)=\exp (-\exp (-x)):=\Lambda(x)
$$

with the normalizing constants $\alpha_{n}$ and $\beta_{n}$ given by

$$
\alpha_{n}=\frac{\sigma}{(2 \log n)^{\frac{1}{2}}}, \quad \beta_{n}=\left(2 \sigma^{2} \log n\right)^{\frac{1}{2}}+\frac{\sigma \log (2 \log n)+\sigma \log \frac{2}{\pi}}{2(2 \log n)^{\frac{1}{2}}} .
$$


By arguments similar to those of Hall [6], Peng et al. [7] and Lin et al. [9], the appropriate normalizing constants $a_{n}$ and $b_{n}$ can be given by the following equations:

$$
a_{n}=\sigma^{2} b_{n}^{-1}
$$

and

$$
\sqrt{\frac{\pi}{2}} \frac{b_{n}}{\sigma} \exp \left(\frac{b_{n}^{2}}{2 \sigma^{2}}\right)=n
$$

By arguments similar to those of Example 2 of Resnick [11], we have

$$
b_{n}=\left(2 \sigma^{2} \log n\right)^{\frac{1}{2}}+\frac{\sigma \log (2 \log n)+\sigma \log \frac{2}{\pi}}{2(2 \log n)^{\frac{1}{2}}}+o\left((\log n)^{-1 / 2}\right) .
$$

Hence

$$
\alpha_{n} / a_{n} \rightarrow 1, \quad\left(\beta_{n}-b_{n}\right) / a_{n} \rightarrow 0,
$$

implying

$$
\lim _{n \rightarrow \infty} P\left(M_{n} \leq a_{n} x+b_{n}\right)=\lim _{n \rightarrow \infty} F^{n}\left(a_{n} x+b_{n}\right)=\Lambda(x),
$$

cf. Leadbetter et al. [12] or Resnick [11].

This paper is organized as follows. Section 2 gives some auxiliary results. In Section 3, we present the main result. Related proofs are given in Section 4.

\section{Auxiliary results}

To establish the uniform convergence of $F^{n}\left(a_{n} x+b_{n}\right)$ to its extreme value distribution $\Lambda(x)$, we need some auxiliary results. The first result is the decomposition of $F(x)$, which is the following result.

Lemma 1 Let $F(x)$ be the Maxwell distribution function. Then, for $x>0$, we have

$$
1-F(x)=\sqrt{\frac{2}{\pi}} \frac{x}{\sigma}\left(1+\frac{\sigma^{2}}{x^{2}}\right) \exp \left(-\frac{x^{2}}{2 \sigma^{2}}\right)-r(x)
$$

with

$$
0<r(x)=\sqrt{\frac{2}{\pi}} \int_{x}^{\infty} \frac{\sigma}{y^{2}} \exp \left(-\frac{y^{2}}{2 \sigma^{2}}\right) d y<\sqrt{\frac{2}{\pi}} \frac{\sigma^{3}}{x^{3}} \exp \left(-\frac{x^{2}}{2 \sigma^{2}}\right) .
$$

For simplicity, throughout this paper, let $C$ be a generic positive constant whose value may change from line to line, and let $C_{i}, C_{i j}(i \in N, j \in N)$ be absolute positive constants.

For the normalizing constants $a_{n}, b_{n}$ defined by (1.3) and (1.4), respectively, let

$$
a_{n}^{*}=a_{n} r_{n}, \quad b_{n}^{*}=b_{n}+a_{n} \delta_{n},
$$

where $r_{n} \rightarrow 1, \delta_{n} \rightarrow 0, n \rightarrow \infty$. So, $a_{n}^{*} / a_{n} \rightarrow 1,\left(b_{n}^{*}-b_{n}\right) / a_{n} \rightarrow 0$, implying $F^{n}\left(a_{n}^{*} x+b_{n}^{*}\right) \rightarrow$ $\Lambda(x)$. For large $n$, we have the following result. 
Lemma 2 Let $a_{n}^{*}, b_{n}^{*}$ be defined by (2.3). For fixed $x \in R$ and sufficiently large $n$, we have

$$
\begin{aligned}
F^{n}\left(a_{n}^{*} x+b_{n}^{*}\right)-\Lambda(x)= & \Lambda(x) e^{-x}\left(\left(\frac{x^{2}}{2}-x-1\right) a_{n} b_{n}^{-1}+\left(r_{n}-1\right) x\right. \\
& \left.+\delta_{n}+O\left[\left(a_{n} b_{n}^{-1}\right)^{2}+\left(r_{n}-1\right)^{2}+\delta_{n}^{2}\right]\right) .
\end{aligned}
$$

Proof Note that $b_{n} \sim \sigma(2 \log n)^{\frac{1}{2}}$, which means

$$
a_{n} b_{n}^{-1} \sim \frac{1}{2 \log n} \rightarrow 0
$$

For large $n$, we have

$$
\begin{aligned}
& \sqrt{\frac{2}{\pi}} \frac{a_{n}^{*} x+b_{n}^{*}}{\sigma} \exp \left(-\frac{\left(a_{n}^{*} x+b_{n}^{*}\right)^{2}}{2 \sigma^{2}}\right) \\
& =\sqrt{\frac{2}{\pi} \frac{b_{n}}{\sigma}}\left(1+a_{n} b_{n}^{-1}\left(r_{n} x+\delta_{n}\right)\right) \exp \left(-\frac{b_{n}^{2}}{2 \sigma^{2}}\right) \\
& \quad \times \exp \left(-\frac{a_{n}^{2}\left(r_{n}^{2} x^{2}+\delta_{n}^{2}+2 r_{n} \delta_{n} x\right)}{2 \sigma^{2}}-\left(r_{n}-1\right) x-x-\delta_{n}\right) \\
& =n^{-1} e^{-x}\left(1-\left(\frac{x^{2}}{2}-x\right) a_{n} b_{n}^{-1}-\left(r_{n}-1\right) x-\delta_{n}+O\left[\left(a_{n} b_{n}^{-1}\right)^{2}+\left(r_{n}-1\right)^{2}+\delta_{n}^{2}\right]\right) .
\end{aligned}
$$

Since

$$
\frac{\sigma^{2}}{\left(a_{n}^{*} x+b_{n}^{*}\right)^{2}}=a_{n} b_{n}^{-1}-2 x\left(a_{n} b_{n}^{-1}\right)^{2}+O\left(\left(a_{n} b_{n}^{-1}\right)^{3}\right)
$$

we have

$$
\frac{\sigma^{4}}{\left(a_{n}^{*} x+b_{n}^{*}\right)^{4}}=\left(a_{n} b_{n}^{-1}\right)^{2}+O\left(\left(a_{n} b_{n}^{-1}\right)^{3}\right) .
$$

Similarly,

$$
\frac{\sigma^{5}}{\left(a_{n}^{*} x+b_{n}^{*}\right)^{5}} \exp \left(-\frac{\left(a_{n}^{*} x+b_{n}^{*}\right)^{2}}{2 \sigma^{2}}\right)=O\left(n^{-1}\left(a_{n} b_{n}^{-1}\right)^{2}\right) \text {. }
$$

Hence,

$$
\begin{aligned}
1-F\left(a_{n}^{*} x+b_{n}^{*}\right)= & n^{-1} e^{-x}\left(1-\left(\frac{x^{2}}{2}-x-1\right) a_{n} b_{n}^{-1}-\left(r_{n}-1\right) x\right. \\
& \left.-\delta_{n}+O\left[\left(a_{n} b_{n}^{-1}\right)^{2}+\left(r_{n}-1\right)^{2}+\delta_{n}^{2}\right]\right) .
\end{aligned}
$$

So,

$$
\begin{aligned}
& F^{n}\left(a_{n}^{*} x+b_{n}^{*}\right)-\Lambda(x) \\
& \quad=\left(1-n^{-1} e^{-x}\left(1-\left(\frac{x^{2}}{2}-x-1\right) a_{n} b_{n}^{-1}-\left(r_{n}-1\right) x\right.\right.
\end{aligned}
$$




$$
\begin{aligned}
& \left.\left.-\delta_{n}+O\left[\left(a_{n} b_{n}^{-1}\right)^{2}+\left(r_{n}-1\right)^{2}+\delta_{n}^{2}\right]\right)\right)^{n}-\Lambda(x) \\
= & \Lambda(x) e^{-x}\left(\left(\frac{x^{2}}{2}-x-1\right) a_{n} b_{n}^{-1}+\left(r_{n}-1\right) x+\delta_{n}+O\left[\left(a_{n} b_{n}^{-1}\right)^{2}+\left(r_{n}-1\right)^{2}+\delta_{n}^{2}\right]\right),
\end{aligned}
$$

which is the desired result.

\section{Main results}

In this section we present the pointwise convergence rate and the uniform convergence rate of $F^{n}(\cdot)$ to its extreme value distribution under different normalizing constants. The first result is the pointwise convergence of extremes under the normalizing constants given by (1.2).

Theorem 1 Let $\left\{\xi_{n}, n \geq 1\right\}$ be a sequence of independent identically distributed random variables with common distribution $M D$. Then

$$
F^{n}\left(\alpha_{n} x+\beta_{n}\right)-\Lambda(x) \sim \Lambda(x) e^{-x} \frac{(\log (2 \log n))^{2}}{16 \log n},
$$

for large $n$, where $\alpha_{n}, \beta_{n}$ are defined in (1.2).

Recently Liu and $\mathrm{Fu}[10]$ proved the result, we present an improved proof for the pointwise convergence rate in Section 4.

The following is the uniform convergence rate of extremes under the appropriate normalizing constants $a_{n}$ and $b_{n}$ given by (1.3) and (1.4), which shows that the optimal convergence rate is proportional to $1 / \log n$.

Theorem 2 Let $\left(\xi_{n}, n \geq 1\right)$ be a sequence of independent identically distributed random variables with common distribution $M D$. For large $n$, there exist absolute constants $0<$ $d_{1}<d_{2}$ such that

$$
\frac{d_{1}}{\log n}<\sup _{x \in R}\left|F^{n}\left(a_{n} x+b_{n}\right)-\Lambda(x)\right|<\frac{d_{2}}{\log n},
$$

where $a_{n}$ and $b_{n}$ are defined by (1.3) and (1.4), respectively.

\section{Proofs}

Proof of Theorem 1 Firstly, we derive the following asymptotic expansions of $b_{n}$ defined by (1.4)

$$
b_{n}=\beta_{n}+o\left((\log n)^{-\frac{1}{2}}\right)
$$

and

$$
\begin{aligned}
b_{n}= & \beta_{n}-\frac{\sigma\left(\log (2 \log n)+\log \frac{2}{\pi}\right)^{2}}{16 \sqrt{2}(\log n)^{\frac{3}{2}}} \\
& +\left(\sigma \log \frac{4 \log n+\log (2 \log n)+\log \frac{2}{\pi}}{4 \log n}\right) /(2 \log n)^{\frac{1}{2}}+O\left(\frac{(\log (2 \log n))^{2}}{(\log n)^{\frac{5}{2}}}\right) .
\end{aligned}
$$


Setting $b_{n}=\beta_{n}+\theta_{n}$ and substituting into (1.4), we obtain by taking logarithms that

$$
\log \frac{\pi}{2}+\log \sigma-\log \left(\beta_{n}+\theta_{n}\right)+\frac{\beta_{n}^{2}}{2 \sigma^{2}}+\frac{\beta_{n} \theta_{n}}{\sigma^{2}}+\frac{\theta_{n}^{2}}{\sigma^{2}}=\log n .
$$

So,

$$
\begin{aligned}
& \frac{\left(\log (2 \log n)+\log \frac{2}{\pi}\right)^{2}}{16 \log n}-\log \frac{4 \log n+\log (2 \log n)+\log \frac{2}{\pi}}{4 \log n} \\
& +\frac{\beta_{n} \theta_{n}}{\sigma^{2}}+\frac{\theta_{n}^{2}}{\sigma^{2}}-\log \left(1+\frac{\theta_{n}}{\beta_{n}}\right)=0
\end{aligned}
$$

therefore

$$
\frac{\beta_{n} \theta_{n}}{\sigma^{2}} \sim-\frac{\left(\log (2 \log n)+\log \frac{2}{\pi}\right)^{2}}{16 \log n}+\log \frac{4 \log n+\log (2 \log n)+\log \frac{2}{\pi}}{4 \log n},
$$

which implies

$$
\begin{aligned}
\theta_{n} \sim- & \frac{\sigma\left(\log (2 \log n)+\log \frac{2}{\pi}\right)^{2}}{16 \sqrt{2}(\log n)^{\frac{3}{2}}} \\
& +\left(\sigma \log \frac{4 \log n+\log (2 \log n)+\log \frac{2}{\pi}}{4 \log n}\right) /(2 \log n)^{\frac{1}{2}} .
\end{aligned}
$$

Once again let

$$
\theta_{n}=-\frac{\sigma\left(\log (2 \log n)+\log \frac{2}{\pi}\right)^{2}}{16 \sqrt{2}(\log n)^{\frac{3}{2}}}+\left(\sigma \log \frac{4 \log n+\log (2 \log n)+\log \frac{2}{\pi}}{4 \log n}\right) /(2 \log n)^{\frac{1}{2}}+v_{n},
$$

where $v_{n}=o\left(\frac{(\log (2 \log n))^{2}}{(\log n)^{\frac{3}{2}}}\right)$. By similar arguments, we can obtain (4.2).

Note that $a_{n}=\frac{\sigma^{2}}{b_{n}}$, we have

$$
r_{n}-1=\frac{\alpha_{n}}{a_{n}}-1 \sim \frac{\log (2 \log n)}{4 \log n}, \quad \delta_{n}=\frac{\beta_{n}-b_{n}}{a_{n}} \sim \frac{(\log (2 \log n))^{2}}{16 \log n} .
$$

Noting $a_{n} b_{n}^{-1} \sim \frac{1}{2 \log n}$, by Lemma 2 , we have

$$
F^{n}\left(\alpha_{n} x+\beta_{n}\right)-\Lambda(x) \sim \Lambda(x) e^{-x} \frac{(\log (2 \log n))^{2}}{16 \log n}
$$

The proof is complete.

Proof of Theorem 2 Letting $r_{n}=1, \delta_{n}=0$ in (2.3) and noting $a_{n} b_{n}^{-1} \sim \frac{1}{2 \log n}$, and by Lemma 2, there exists an absolute constant $d_{1}>0$ such that

$$
\sup _{x \in R}\left|F^{n}\left(a_{n} x+b_{n}\right)-\Lambda(x)\right|>\frac{d_{1}}{\log n} .
$$


Thus, in order to obtain the upper bound, we need to prove
(a) $\sup _{-c_{n} \leq x<0}\left|F^{n}\left(a_{n} x+b_{n}\right)-\Lambda(x)\right|<\mathbb{D}_{1} a_{n} b_{n}^{-1}$,
(b) $\sup _{0 \leq x \leq d_{n}}\left|F^{n}\left(a_{n} x+b_{n}\right)-\Lambda(x)\right|<\mathbb{D}_{2} a_{n} b_{n}^{-1}$,
(c) $\sup _{d_{n} \leq x<\infty}\left|F^{n}\left(a_{n} x+b_{n}\right)-\Lambda(x)\right|<\mathbb{D}_{3} a_{n} b_{n}^{-1}$,
(d) $\sup _{-\infty<x \leq-c_{n}}\left|F^{n}\left(a_{n} x+b_{n}\right)-\Lambda(x)\right|<\mathbb{D}_{4} a_{n} b_{n}^{-1}$,

where $\mathbb{D}_{i}>0(i=1,2,3,4)$, and

$$
c_{n}=: \log \log \frac{b_{n}^{2}}{\sigma^{2}}>0, \quad d_{n}=:-\log \log \frac{b_{n}^{2}}{b_{n}^{2}-\sigma^{2}}>0 .
$$

Obviously,

$$
\sigma(2 \log n)^{\frac{1}{2}}<b_{n}<\sigma(2 \log n)^{\frac{1}{2}}\left(1+C_{0}\right)
$$

and

$$
b_{n}-a_{n} c_{n}=b_{n}\left(1-\frac{\sigma^{2}}{b_{n}^{2}} c_{n}\right)=b_{n}\left(1-\frac{\sigma^{2}}{b_{n}^{2}} \log \log \frac{b_{n}^{2}}{\sigma^{2}}\right)>0 .
$$

Define $\Psi_{n}(x)=1-F\left(a_{n} x+b_{n}\right)$, then

$$
\begin{aligned}
n \log \left(1-F\left(a_{n} x+b_{n}\right)\right) & =-n \Psi_{n}(x)+n \Psi_{n}(x)+n \log \left(1-\Psi_{n}(x)\right) \\
& =-n \Psi_{n}(x)-R_{n}(x) .
\end{aligned}
$$

By the following inequality

$$
-x-\frac{x^{2}}{2(1-x)}<\log (1-x)<-x \quad(0<x<1),
$$

we have

$$
0<R_{n}(x)=-\left(n \Psi_{n}(x)+n \log \left(1-\Psi_{n}(x)\right)\right)<\frac{n \Psi_{n}^{2}(x)}{2\left(1-\Psi_{n}(x)\right)} .
$$

First, suppose that $x \geq-c_{n}$. By (2.1), we have

$$
\begin{aligned}
\Psi_{n}(x) & \leq \Psi_{n}\left(-c_{n}\right)=1-F\left(b_{n}-a_{n} c_{n}\right) \\
& <\sqrt{\frac{2}{\pi}} \frac{b_{n}-a_{n} c_{n}}{\sigma}\left(1+\frac{\sigma^{2}}{\left(b_{n}-a_{n} c_{n}\right)^{2}}\right) \exp \left(-\frac{\left(b_{n}-a_{n} c_{n}\right)^{2}}{2 \sigma^{2}}\right) \\
& <2 \sqrt{\frac{2}{\pi}} \frac{b_{n}}{\sigma}\left(1-a_{n} b_{n}^{-1} c_{n}\right) \exp \left(-\frac{\left(b_{n}\right)^{2}}{2 \sigma^{2}}+c_{n}-\frac{a_{n} b_{n}^{-1} c_{n}^{2}}{2}\right) \\
& <2 n^{-1} e^{c_{n}}=2 n^{-1} \log \frac{b_{n}^{2}}{\sigma^{2}} \\
& <\sup _{n \geq n_{0}} \frac{2 \log \left(C_{1} \log n\right)}{n}<\mathfrak{C}_{11}<1
\end{aligned}
$$


with $C_{1}=2\left(1+C_{0}\right)^{2}$, implying

$$
\inf _{x \geq-c_{n}}\left(1-\Psi_{n}(x)\right)>1-\mathfrak{C}_{11}>0 .
$$

Therefore,

$$
\begin{aligned}
0 & <R_{n}(x) \leq \frac{n \Psi_{n}^{2}(x)}{2\left(1-\mathfrak{C}_{11}\right)} \leq \frac{n \Psi_{n}^{2}\left(-c_{n}\right)}{2\left(1-\mathfrak{C}_{11}\right)} \\
& <\frac{n^{-1}\left(\log \left(C_{1} \log n\right)\right)^{2}}{2\left(1-\mathfrak{C}_{11}\right)}=\frac{n^{-1}\left(\log \left(C_{1} \log n\right)\right)^{2} a_{n} b_{n}^{-1}}{2\left(1-\mathfrak{C}_{11}\right) a_{n} b_{n}^{-1}} \\
& <\frac{n^{-1}\left(\log \left(C_{1} \log n\right)\right)^{2}}{4\left(1-\mathfrak{C}_{11}\right) \log n} a_{n} b_{n}^{-1} \\
& <C a_{n} b_{n}^{-1} .
\end{aligned}
$$

By $1-e^{-x}<x, x>0$, we have

$$
\left|\exp \left(-R_{n}(x)\right)-1\right|<R_{n}(x)<C a_{n} b_{n}^{-1} .
$$

Setting $A_{n}(x)=\exp \left(-n \Psi_{n}(x)+e^{-x}\right), B_{n}(x)=\exp \left(-R_{n}(x)\right)$, we obtain

$$
\begin{aligned}
\left|F^{n}\left(a_{n} x+b_{n}\right)-\Lambda(x)\right| & =\Lambda(x)\left|A_{n}(x) B_{n}(x)-1\right| \\
& =\Lambda(x)\left|A_{n}(x) B_{n}(x)-B_{n}(x)+B_{n}(x)-1\right| \\
& \leq \Lambda(x)\left|A_{n}(x)-1\right|+\left|B_{n}(x)-1\right| \\
& <\Lambda(x)\left|A_{n}(x)-1\right|+C a_{n} b_{n}^{-1} .
\end{aligned}
$$

By (2.1) and (2.2), we have

$$
\begin{aligned}
-n \Psi_{n}(x)+e^{-x}= & -n\left[\sqrt{\frac{2}{\pi}} \frac{b_{n}+a_{n} x}{\sigma}\left(1+\frac{\sigma^{2}}{\left(b_{n}+a_{n} x\right)^{2}}\right) \exp \left(-\frac{\left(b_{n}+a_{n} x\right)^{2}}{2 \sigma^{2}}\right)\right. \\
& \left.-r\left(a_{n} x+b_{n}\right)\right]+e^{-x} \\
= & \left(1+a_{n} b_{n}^{-1} x\right) e^{-x} C_{n}(x),
\end{aligned}
$$

where

$$
C_{n}(x)=\left(-1-\frac{\sigma^{2}}{\left(b_{n}+a_{n} x\right)^{2}}+\frac{\sigma^{4}}{\left(b_{n}+a_{n} x\right)^{4}} \delta_{n}\left(a_{n} x+b_{n}\right)\right) \exp \left(-\frac{a_{n} b_{n}^{-1} x^{2}}{2}\right)+\left(1+a_{n} b_{n}^{-1} x\right)^{-1}
$$

with $0<\delta_{n}\left(a_{n} x+b_{n}\right)<1$. To prove (4.7), we consider the case of $-c_{n} \leq x<0$. By $e^{-x}>1-x$, $x>0$, we have

$$
\begin{aligned}
C_{n}(x) & <\left(1-\frac{a_{n} b_{n}^{-1} x^{2}}{2}\right)\left(\left(-1+\frac{\sigma^{4}}{\left(b_{n}+a_{n} x\right)^{4}}\right) \delta_{n}\left(a_{n} x+b_{n}\right)\right)+\left(1+a_{n} b_{n}^{-1} x\right)^{-1} \\
& <\left(1-\frac{a_{n} b_{n}^{-1} x^{2}}{2}\right)\left\{-1+\left(a_{n} b_{n}^{-1}\right)^{2}\left(1+a_{n} b_{n}^{-1} x\right)^{-4}\right\}+\left(1+a_{n} b_{n}^{-1} x\right)^{-1} \\
& =\left(\left(1+a_{n} b_{n}^{-1} x\right)^{-4}+\frac{x^{2}}{2}-x\left(1+a_{n} b_{n}^{-1} x\right)^{-1}\right) a_{n} b_{n}^{-1}
\end{aligned}
$$


and

$$
\begin{aligned}
C_{n}(x) & >\left(-1-\frac{\sigma^{2}}{\left(b_{n}+a_{n} x\right)^{2}}\right) \exp \left(-\frac{a_{n} b_{n}^{-1} x^{2}}{2}\right)+\left(1+a_{n} b_{n}^{-1} x\right)^{-1} \\
& >\left(-1-\frac{\sigma^{2}}{\left(b_{n}+a_{n} x\right)^{2}}\right)+\left(1+a_{n} b_{n}^{-1} x\right)^{-1} \\
& >\left(-\left(1+a_{n} b_{n}^{-1} x\right)^{-2}-x\left(1+a_{n} b_{n}^{-1} x\right)^{-1}\right) a_{n} b_{n}^{-1} \\
& >-\left(1+a_{n} b_{n}^{-1} x\right)^{-2} .
\end{aligned}
$$

Hence, for $-c_{n} \leq x<0$, by combining (4.13) and (4.14) together, we have

$$
\begin{aligned}
\left|C_{n}(x)\right| & <\left(\left(1+a_{n} b_{n}^{-1} x\right)^{-4}+\frac{x^{2}}{2}-x\left(1+a_{n} b_{n}^{-1} x\right)^{-1}+\left(1+a_{n} b_{n}^{-1} x\right)^{-2}\right) a_{n} b_{n}^{-1} \\
& <\left(\left(1-a_{n} b_{n}^{-1} c_{n}\right)^{-4}+\frac{c_{n}^{2}}{2}+c_{n}\left(1-a_{n} b_{n}^{-1} c_{n}\right)^{-1}+\left(1-a_{n} b_{n}^{-1} c_{n}\right)^{-2}\right) a_{n} b_{n}^{-1} \\
& <C_{21} .
\end{aligned}
$$

Furthermore, for $-c_{n} \leq x<0$, we have

$$
\begin{aligned}
\left|-n \Psi_{n}(x)+e^{-x}\right|< & \left(1+a_{n} b_{n}^{-1} x\right) e^{-x}\left|C_{n}(x)\right| \\
& <\left(\left(1+a_{n} b_{n}^{-1} x\right)^{-4}+\frac{x^{2}}{2}-x\left(1+a_{n} b_{n}^{-1} x\right)^{-1}+\left(1+a_{n} b_{n}^{-1} x\right)^{-2}\right) e^{-x} a_{n} b_{n}^{-1} \\
& <\left(\left(1-a_{n} b_{n}^{-1} c_{n}\right)^{-4}+\frac{c_{n}^{2}}{2}+c_{n}\left(1-a_{n} b_{n}^{-1} c_{n}\right)^{-1}\right. \\
& \left.+\left(1-a_{n} b_{n}^{-1} c_{n}\right)^{-2}\right) e^{c_{n}} a_{n} b_{n}^{-1} \\
& <C_{22} .
\end{aligned}
$$

Noting that $0<\left|e^{x}-1\right|<|x|\left(e^{x}+1\right), x \in R$ and $e^{-x}>1-x+x^{2} / 2$ for $-c_{n} \leq x<0$, we have

$$
\begin{aligned}
\Lambda(x)\left|A_{n}(x)-1\right|= & \Lambda(x)\left|\exp \left(-n \Psi_{n}(x)+e^{-x}\right)-1\right| \\
< & \Lambda(x)\left|-n \Psi_{n}(x)+e^{-x}\right|\left(\exp \left(-n \Psi_{n}(x)+e^{-x}\right)+1\right) \\
< & \left(e^{C_{22}}+1\right)\left(\left(1+a_{n} b_{n}^{-1} x\right)^{-4}+\frac{x^{2}}{2}-x\left(1+a_{n} b_{n}^{-1} x\right)^{-1}+\left(1+a_{n} b_{n}^{-1} x\right)^{-2}\right) \\
& \times a_{n} b_{n}^{-1} \exp \left(-e^{-x}-x\right) \\
< & C_{23} a_{n} b_{n}^{-1} .
\end{aligned}
$$

Together with (4.12), we establish (4.7).

Second, we prove (4.8). Note that

$$
\begin{aligned}
C_{n}(x) & <\left(-1+\frac{\sigma^{4}}{\left(b_{n}+a_{n} x\right)^{4}} \delta_{n}\left(a_{n} x+b_{n}\right)\right)\left(1-\frac{a_{n} b_{n}^{-1} x^{2}}{2}\right)+\left(1+a_{n} b_{n}^{-1} x\right)^{-1} \\
& <\left(a_{n} b_{n}^{-1}\right)^{2}\left(1+a_{n} b_{n}^{-1} x\right)^{-4}+\frac{x^{2}}{2} a_{n} b_{n}^{-1}-a_{n} b_{n}^{-1} x\left(1+a_{n} b_{n}^{-1} x\right)^{-1}
\end{aligned}
$$




$$
\begin{aligned}
& <\left(a_{n} b_{n}^{-1}\right)^{2}\left(1+a_{n} b_{n}^{-1} x\right)^{-4}+\frac{x^{2}}{2} a_{n} b_{n}^{-1} \\
& <\left(1+\frac{x^{2}}{2}\right) a_{n} b_{n}^{-1}
\end{aligned}
$$

and

$$
C_{n}(x)>\left(-\left(1+a_{n} b_{n}^{-1} x\right)^{-2}-x\left(1+a_{n} b_{n}^{-1} x\right)^{-1}\right) a_{n} b_{n}^{-1} .
$$

By (4.15) and (4.16), for $0 \leq x<d_{n}$, we have

$$
\begin{aligned}
\left|C_{n}(x)\right| & <\left(1+\frac{x^{2}}{2}+\left(1+a_{n} b_{n}^{-1} x\right)^{-2}+x\left(1+a_{n} b_{n}^{-1} x\right)^{-1}\right) a_{n} b_{n}^{-1} \\
& <\left(2+x+\frac{x^{2}}{2}\right) a_{n} b_{n}^{-1} .
\end{aligned}
$$

Hence,

$$
\begin{aligned}
\left|-n \Psi_{n}(x)+e^{-x}\right| & <\left(1+a_{n} b_{n}^{-1} x\right) e^{-x}\left|C_{n}(x)\right| \\
& <\left(1+a_{n} b_{n}^{-1} x\right) e^{-x}\left(2+x+\frac{x^{2}}{2}\right) a_{n} b_{n}^{-1} \\
& <C_{31} a_{n} b_{n}^{-1}<C_{32} .
\end{aligned}
$$

Therefore

$$
\begin{aligned}
\Lambda(x)\left|A_{n}(x)-1\right| & <\Lambda(x)\left|-n \Psi_{n}(x)+e^{-x}\right|\left(\exp \left(-n \Psi_{n}(x)+e^{-x}\right)+1\right) \\
& <C_{31}\left(e^{C_{32}}+1\right) \Lambda\left(d_{n}\right) a_{n} b_{n}^{-1} \\
& <C_{33} a_{n} b_{n}^{-1}
\end{aligned}
$$

Combining (4.12) and (4.17) together, we can derive that

$$
\sup _{0 \leq x \leq d_{n}}\left|F^{n}\left(a_{n} x+b_{n}\right)-\Lambda(x)\right|<\left(C_{12}+C_{33}\right) a_{n} b_{n}^{-1}=: \mathbb{D}_{2} a_{n} b_{n}^{-1}
$$

Hence (4.8) is proved.

Third, for $x \geq d_{n}$, we have

$$
\sup _{x \geq d_{n}}(1-\Lambda(x)) \leq 1-\Lambda\left(d_{n}\right)=a_{n} b_{n}^{-1}
$$

By $1-e^{x}<-x, x \in R$, we have

$$
\begin{aligned}
1-F^{n}\left(a_{n} d_{n}+b_{n}\right) & =1-\exp \left(n \log F\left(a_{n} d_{n}+b_{n}\right)\right) \\
& <-n \log F\left(a_{n} d_{n}+b_{n}\right) \\
& =n \Psi_{n}\left(d_{n}\right)+R_{n}\left(d_{n}\right) .
\end{aligned}
$$


By $(2.1)$ and $\log (1+x)<x, 0<x<1$, we have

$$
\begin{aligned}
n \Psi_{n}\left(d_{n}\right) & =n\left(1-F\left(a_{n} d_{n}+b_{n}\right)\right) \\
& <\left(1+a_{n} b_{n}^{-1} d_{n}\right) e^{-d_{n}}\left(1+a_{n} b_{n}^{-1}\left(1+a_{n} b_{n}^{-1} d_{n}\right)^{-2}\right) \\
& <2\left(1+a_{n} b_{n}^{-1} d_{n}\right) \log \frac{b_{n}^{2}}{b_{n}^{2}-\sigma^{2}} \\
& <2\left(d_{n}+a_{n}^{-1} b_{n}\right) \frac{\sigma^{2}}{b_{n}^{2}-\sigma^{2}} a_{n} b_{n}^{-1} \\
& =\left(1+\frac{\sigma^{2}}{b_{n}^{2}-\sigma^{2}}-\frac{\sigma^{2}}{b_{n}^{2}-\sigma^{2}} \log \log \left(\frac{\sigma^{2}}{b_{n}^{2}-\sigma^{2}}\right)\right) a_{n} b_{n}^{-1} \\
& <C_{41} a_{n} b_{n}^{-1} .
\end{aligned}
$$

Noting that $R_{n}\left(d_{n}\right)<C_{12} a_{n} b_{n}^{-1}$, and combining (4.18), (4.19), (4.20) and (4.14) together, we have

$$
\begin{aligned}
\sup _{x \geq d_{n}}\left|F^{n}\left(a_{n} x+b_{n}\right)-\Lambda(x)\right| & <\sup _{x \geq d_{n}}\left(1-F^{n}\left(a_{n} x+b_{n}\right)\right)+\sup _{x \geq d_{n}}(1-\Lambda(x)) \\
& <n \Psi_{n}\left(d_{n}\right)+R_{n}\left(d_{n}\right)+a_{n} b_{n}^{-1} \\
& <\left(C_{41}+C_{12}+1\right) a_{n} b_{n}^{-1}=: \mathbb{D}_{3} a_{n} b_{n}^{-1},
\end{aligned}
$$

which is (4.9).

Finally, consider the case of $-\infty<x<-c_{n}$. If $a_{n} x+b_{n} \leq 0$, then $F^{n}\left(a_{n} x+b_{n}\right)=0$. By $\Lambda(-x)<\frac{1}{x}, x>1$, we have

$$
\sup _{x \leq-b_{n} / a_{n}}\left|F^{n}\left(a_{n} x+b_{n}\right)-\Lambda(x)\right|=\sup _{x \leq-b_{n} / a_{n}} \Lambda(x) \leq \Lambda\left(-b_{n}^{2} / \sigma^{2}\right)<\frac{\sigma^{2}}{b_{n}^{2}}=a_{n} b_{n}^{-1}
$$

So, we only need to consider the case of $a_{n} x+b_{n}>0$. By using the monotonicity of $\Lambda(x)$, we have

$$
\sup _{x \leq-c_{n}} \Lambda(x) \leq \Lambda\left(-c_{n}\right)=a_{n} b_{n}^{-1}
$$

Noting $\log (1-x)<-x, 0<x<1$ and $e^{-x}>1-x, x \in R$, and combining (2.1) and (2.2) together, we have

$$
\begin{aligned}
\sup _{x \leq-c_{n}} & F^{n}\left(a_{n} x+b_{n}\right) \\
& \leq F^{n}\left(b_{n}-a_{n} c_{n}\right) \\
& <\left(1-n^{-1}\left(1-a_{n} b_{n}^{-1} c_{n}\right)\left(1-\left(a_{n} b_{n}^{-1}\right)^{2}\left(1-a_{n} b_{n}^{-1} c_{n}\right)^{-4}\right) \exp \left(c_{n}-\frac{a_{n} b_{n}^{-1} c_{n}^{2}}{2}\right)\right)^{n} \\
& <\exp \left(-e^{c_{n}}\left(1-a_{n} b_{n}^{-1} c_{n}\right)\left(1-\left(a_{n} b_{n}^{-1}\right)^{2}\left(1-a_{n} b_{n}^{-1} c_{n}\right)^{-4}\right) \exp \left(-\frac{a_{n} b_{n}^{-1} c_{n}^{2}}{2}\right)\right) \\
& <\exp \left(-e^{c_{n}}\left(1-\left(a_{n} b_{n}^{-1} c_{n}+\left(a_{n} b_{n}^{-1}\right)^{2}\left(1-a_{n} b_{n}^{-1} c_{n}\right)^{-3}+\frac{a_{n} b_{n}^{-1} c_{n}^{2}}{2}\right)\right)\right)
\end{aligned}
$$




$$
\begin{aligned}
& <\exp \left(-e^{c_{n}}\right) \exp \left(\left(a_{n} b_{n}^{-1} c_{n}+\left(a_{n} b_{n}^{-1}\right)^{2}\left(1-a_{n} b_{n}^{-1} c_{n}\right)^{-3}+\frac{a_{n} b_{n}^{-1} c_{n}^{2}}{2}\right) e^{c_{n}}\right) \\
& <C_{51} a_{n} b_{n}^{-1} .
\end{aligned}
$$

Together with (4.21), we have

$$
\begin{aligned}
\sup _{-\infty<x \leq-c_{n}}\left|F^{n}\left(a_{n} x+b_{n}\right)-\Lambda(x)\right| & \leq \sup _{-\infty<x<-c_{n}} F^{n}\left(a_{n} x+b_{n}\right)+\sup _{-\infty<x<-c_{n}} \Lambda(x) \\
& \leq F^{n}\left(b_{n}-a_{n} c_{n}\right)+\Lambda\left(-c_{n}\right) \\
& <\left(C_{12}+1\right) a_{n} b_{n}^{-1}=: \mathbb{D}_{4} a_{n} b_{n}^{-1} .
\end{aligned}
$$

This is (4.10). The proof is complete.

\section{Competing interests}

The authors declare that they have no competing interests.

\section{Authors' contributions}

All authors read and approved the final manuscript.

\section{Acknowledgements}

The research was supported by the National Natural Science Foundation of China (11171275) and the Fundamental Research Funds for the Central Universities (XDJK2012C045).

Received: 30 May 2013 Accepted: 1 October 2013 Published: 07 Nov 2013

\section{References}

1. Falk, A: Rate of uniform convergence of extreme order statistics. Ann. Inst. Stat. Math. 38(2), $245-262$ (1986)

2. Balkema, AA, de Haan, L: A convergence rate in extreme-value theory. J. Appl. Probab. 27, 577-585 (1990)

3. de Haan, L, Resnick, SI: Second order regular variation and rates of convergence in extreme value theory. Ann. Probab. 24, 97-124 (1996)

4. Cheng, S, Jiang, C: The Edgeworth expansion for distributions of extreme values. Sci. China Ser. A 4, 427-437 (2001)

5. Hall, WJ, Wellner, JA: The rate of convergence in law of the maximum of an exponential sample. Stat. Neerl. 33 , 151-154 (1979)

6. Hall, P: On the rate of convergence of normal extremes. J. Appl. Probab. 16, 433-439 (1979)

7. Peng, Z, Nadarajah, S, Lin, F: Convergence rate of extremes for the general error distribution. J. Appl. Probab. 47, 668-679 (2010)

8. Lin, F, Peng, Z: Tail behavior and extremes of short-tailed symmetric distribution. Commun. Stat., Theory Methods 39 2811-2817 (2010)

9. Lin, F, Zhang, X, Peng, Z, Jiang, Y: On the rate of convergence of STSD extremes. Commun. Stat., Theory Methods 40 , 1795-1806 (2011)

10. Liu, B, Fu, Y: The pointwise rate of extremes for Maxwell distribution. J. Southwest Univ., Nat. Sci. 5, 86-99 (2013)

11. Resnick, SI: Extreme Values, Regular Variation and Point Processes. Springer, New York (1987)

12. Leadbetter, MR, Lindgren, G, Rootzen, H: Extremes and Related Properties of Random Sequences and Processes. Springer, New York (1983) 